\title{
Identification of SERPINA1 as single marker for papillary thyroid carcinoma through microarray meta analysis and quantification of its discriminatory power in independent validation
}

Klemens Vierlinger ${ }^{1 *}$, Markus H Mansfeld ${ }^{1}$, Oskar Koperek ${ }^{2}$, Christa Nöhammer ${ }^{1}$, Klaus Kaserer ${ }^{2}$ and Friedrich Leisch ${ }^{3}$

\begin{abstract}
Background: Several DNA microarray based expression signatures for the different clinically relevant thyroid tumor entities have been described over the past few years. However, reproducibility of these signatures is generally low, mainly due to study biases, small sample sizes and the highly multivariate nature of microarrays. While there are new technologies available for a more accurate high throughput expression analysis, we show that there is still a lot of information to be gained from data deposited in public microarray databases. In this study we were aiming (1) to identify potential markers for papillary thyroid carcinomas through meta analysis of public microarray data and (2) to confirm these markers in an independent dataset using an independent technology.
\end{abstract}

Methods: We adopted a meta analysis approach for four publicly available microarray datasets on papillary thyroid carcinoma (PTC) nodules versus nodular goitre (NG) from N2-frozen tissue. The methodology included merging of datasets, bias removal using distance weighted discrimination (DWD), feature selection/inference statistics, classification/crossvalidation and gene set enrichment analysis (GSEA). External Validation was performed on an independent dataset using an independent technology, quantitative RT-PCR (RT-qPCR) in our laboratory.

Results: From meta analysis we identified one gene (SERPINA1) which identifies papillary thyroid carcinoma against benign nodules with $99 \%$ accuracy ( $n=99$, sensitivity $=0.98$, specificity $=1$, PPV $=1, N P V=0.98$ ). In the independent validation data, which included not only PTC and NG, but all major histological thyroid entities plus a few variants, SERPINA1 was again markedly up regulated (36-fold, $p=1: 3^{* 1} 10^{-10}$ ) in PTC and identification of papillary carcinoma was possible with $93 \%$ accuracy $(n=82$, sensitivity $=1$, specificity $=0.90, P P V=0.76, N P V=1)$. We also show that the extracellular matrix pathway is strongly activated in the meta analysis data, suggesting an important role of tumor-stroma interaction in the carcinogenesis of papillary thyroid carcinoma.

Conclusions: We show that valuable new information can be gained from meta analysis of existing microarray data deposited in public repositories. While single microarray studies rarely exhibit a sample number which allows robust feature selection, this can be achieved by combining published data using DWD. This approach is not only efficient, but also very cost-effective. Independent validation shows the validity of the results from this meta analysis and confirms SERPINA1 as a potent mRNA marker for PTC in a total (meta analysis plus validation) of 181 samples.

\footnotetext{
* Correspondence: klemens.vierlinger@ait.ac.at

${ }^{1}$ Molecular Medicine, AIT - Austrian Institute of Technology, A-1190 Vienna,

Austria

Full list of author information is available at the end of the article
} 


\section{Background}

Thyroid nodules are endemic in iodine deficient areas, like Europes alpine regions, where they have a prevalence of $10-20 \%$. They are classified by their histology into five main classes: the benign types Nodular Goiter (NG) and Follicular Thyroid Adenoma (FTA), and the malignant entities Follicular Thyroid Carcinoma (FTC), Papillary Thyroid Carcinoma (PTC), Medullary Thyroid Carcinoma (MTC) and Anaplastic Thyroid Carcinoma (ATC). Only approximately $5 \%-10 \%$ of thyroid nodules are malignant [1], the majority of which are papillary carcinomas. Conventionally, discrimination between benign and malignant thyroid nodules is attempted by fine needle aspiration biopsy (FNAB) followed by cytological assessment. Despite many advances in the diagnosis and treatment of thyroid nodules and thyroid cancer, these methods have a well known low specificity [2], resulting in an 'indeterminate' or 'suspicious' diagnosis in $10 \%-20 \%$ of cases. These patients usually undergo surgery, although in only $20 \%$ of these cases the nodules are actually malignant $[3,4]$. This leads to a number of patients unnecessarily treated for malignant disease.

In other types of cancer it has been shown that gene expression profiling can add substantial value to the discrimination of the different clinically relevant tumorentities $[5,6]$. To date, many studies have tried to classify the different entities of thyroid carcinoma on the basis of their gene expression profiles. Each study has published a gene list which they believe discriminates between benign and malignant thyroid nodules or between different tumor entities. However, the lists have no or very few genes in common and applying a classifier from one study to the data from another study generally yields poor classification results. A notable exception to this are the studies from Jarzab et al. and Eszlinger et al. $[7,8]$ who established a 20-gene signature for PTC which they were able to apply to another study and classify all samples correctly.

Here we focus on Papillary Thyroid Carcinoma (PTC), which is the most common and therefore most extensively studied form of thyroid cancer. We hypothesised, that feature selection based on a larger sample cohort will be more robust than using single studies, so we decided for a meta analysis approach which allows us to analyse all publicly avail-able datasets (99 samples) for PTC with one common analysis approach. From this dataset we were able to identify SERPINA1 as a single gene which allows to discriminate between PTC and benign nodules or healthy tissue with $99 \%$ accuracy (one misclassification). To validate these findings and to quantify the discriminatory power of SERPINA1 for the detection of PTC, we performed RT-qPCR experiments on an independent set of thyroid nodules (instantaneous sections) measuring the mRNA levels of SERPINA1. In contrast to the meta analysis data, we included not only PTC and benign nodules but also all other histological entities of thyroid nodules plus rare histological variants (follicular variant and tall cell PTC). Despite the marked overrepresentation of these difficult to diagnose histological variants, the classification accuracy was still as high as $93 \%$.

Encouraged by the high classification accuracy in the independent validation data, showing the validity of our meta analysis approach, we went on to perform Gene Set Enrichment Analysis (GSEA) on the meta-analysis microarray data to elucidate some of the specific mechanisms involved in papillary thyroid tumorigenesis.

\section{Results}

\section{Data Integration}

Data Integration was performed using Distance Weighted Discrimination (DWD) [9]. Figure 1 shows the effect of DWD integration on the first two principal components (PC) and on hierarchical clustering. DWD removes the dataset bias very efficiently while preserving the biological information. Discretisation methods and bayesian methods were not able to remove the clustering by dataset in PCA or hierarchical clustering (data not shown).

\section{Classification}

First, and as a quality measure for each study, each dataset was taken separately (before DWD-integration) and a pamr classification and leave-one-out cross-validation (loocv) was performed. The results of the cross-validations are near perfect with single samples classifying wrongly. However, with the exception of the classifier from the $\mathrm{He}$ dataset, none of these classifiers can be applied to any of the other datasets. Classification results are rarely ever higher than expected by chance. If, however, one uses the DWD-integrated data, the classifiers already fit much better (see Table 1).

Then a pamr - classifier for the distinction of PTC versus various types of benign thyroid tissue was built for the complete DWD-integrated dataset and validated in a leave-one-out crossvalidation. This identified a one gene classifier, which classifies $99 \%$ of samples correctly in leave-one-out-crossvalidation (loocv). The discriminative gene is SERPINA1. If one removes the SERPINA1-probe from the analysis, pamr again finds a classifier with $99 \%$ accuracy in loocv, this time using a 9-gene signature. Removing these 9 genes yields another 9-gene classifier with a similar performance ( $99 \%$ accuracy), and further an 11-gene classifier with $99 \%$ accuracy. In all of these cases, the same sample is misclassified. The expression profile of these genes is visualised in the Figure 2. 


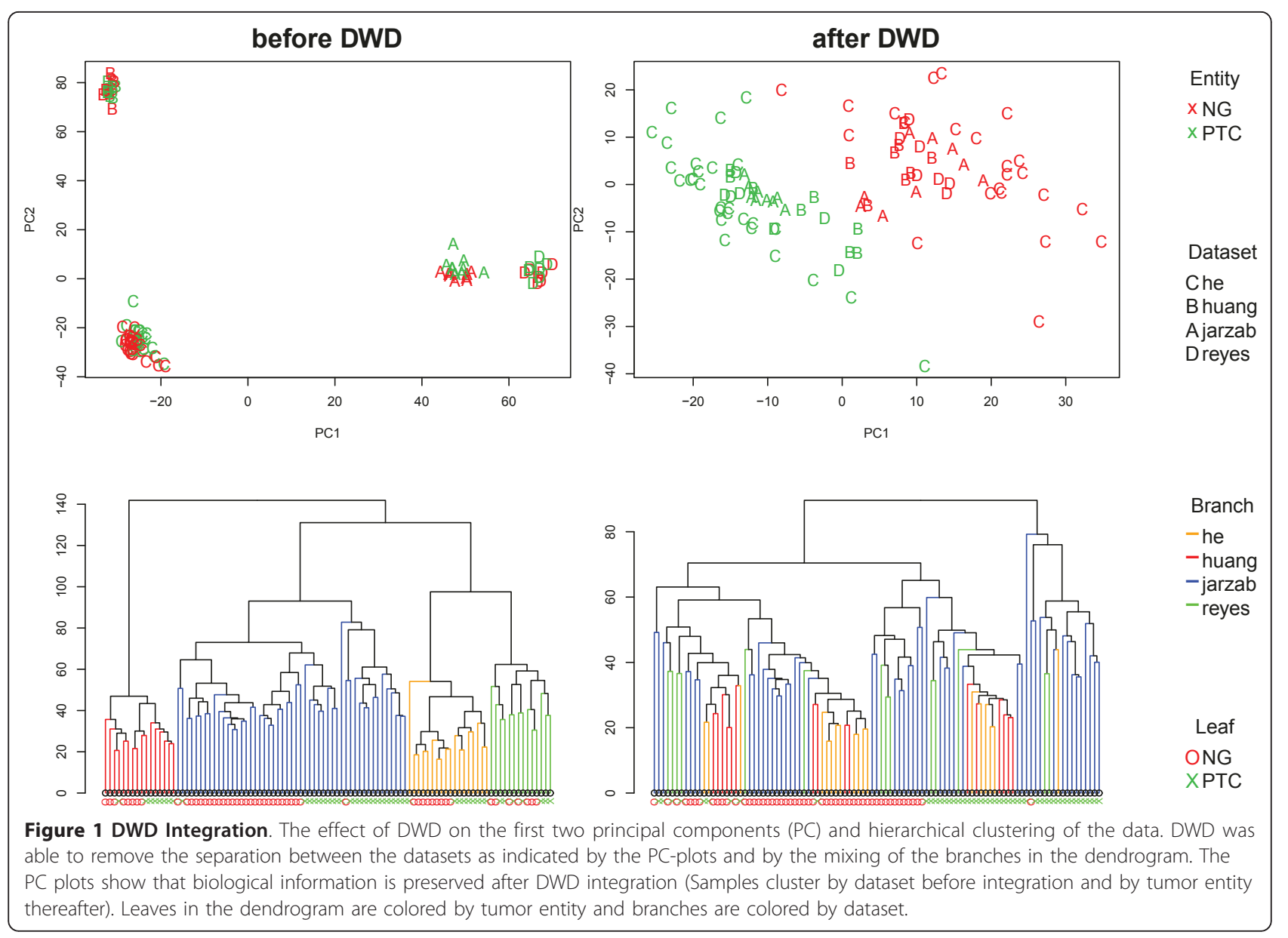

\section{qPCR validation}

Next, we used RT-qPCR to test the discriminative power of SERPINA1 on an independent dataset generated in our own laboratories. Figure 3 shows the SERPINA1 gene expression and ROC analysis in the meta analysis across different studies and in the RT-qPCR data across the different entities. The upregulation of SERPINA1 in the meta analysis data is 8 -fold ( $\mathrm{p}<2$ * $\left.10^{-16}\right)$ and 36 -fold $\left(\mathrm{p}=1.3 * 10^{-10}\right)$ in the $\mathrm{qPCR}$ data (graphs are on a $\log 2$-scale). In the meta analysis data SERPINA1 expression accurately distinguishes PTC

Table 1 Classification Results before and after DWD integration

\begin{tabular}{lcccccccc}
\hline & & before & DWD & & & after & DWD & \\
\hline $\begin{array}{l}\text { test } \rightarrow \\
\text { train } \downarrow\end{array}$ & he & huang & jarzab & reyes & he & huang & jarzab & reyes \\
\hline he & 1.00 & 1.00 & 0.98 & 1.00 & 1.00 & 1.00 & 0.96 & 1.00 \\
huang & 0.50 & 1.00 & 0.55 & 0.50 & 0.50 & 1.00 & 0.90 & 0.71 \\
jarzab & 0.50 & 0.81 & 1.00 & 0.57 & 0.89 & 1.00 & 1.00 & 1.00 \\
reyes & 0.78 & 0.50 & 0.92 & 1.00 & 0.89 & 0.88 & 0.90 & 1.00 \\
\hline
\end{tabular}

Classification results for PTC-data when applying classifiers from one study on another study. Before (left) and after (right) DWD integration. from benign nodules for $99 \%$ of the cases. Note that this is the DWD-integrated data. In the independent validation RT-qPCR data, where we picked samples from all histological entities, the papillary carcinomas again show a distinctly elevated expression of SERPINA1 compared to all other histological groups under investigation. A threshold value of 1.08 (SERPINA1 expression, normalised by DAD1-expression) was used as decision boundary for the decision PTC vs nonPTC. At this threshold, PTC can be detected with a sensitivity of 1 and a specificity of 0.905 (93\% prediction accuracy). False Positive were 2 MTCs, 1 ATC, 2 FTCs (1 recurrent) and 1 FTA (oxyphilic). The meta analysis data classified one PTC falsely as benign, all others were correct.

\section{Differential Expression Analysis and Gene Set Enrichment}

Encouraged by the high reproducibility of the meta analysis results in the external validation data, we went on to perform inference statistics on the meta analysis data. Table 2 shows the 20 most significant differentially expressed genes from inference statistics. SERPINA1 is the most differentially expressed gene, but there are many other genes which are also highly significant. 


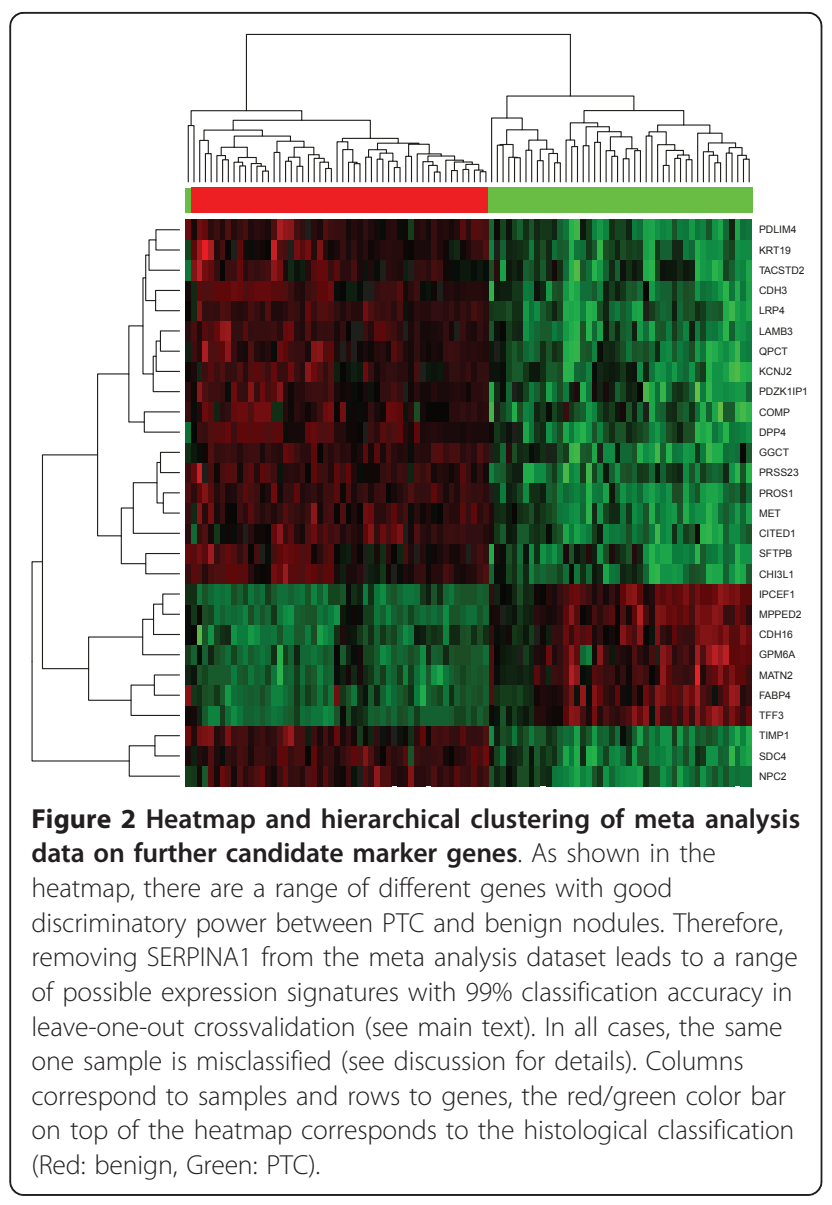

Amongst the highly significant genes, most are upregulated. With a $\log 2$-ratio of 3.3 (corresponds to a 10-fold upregulation), SERPINA1 is not only the most significant gene, but also the gene with the largest effect size. We also used the results from inference statistics for GSEA with the goal of finding enriched gene sets, i.e. gene sets which have higher accumulated t-statistics than expected by chance [10]. In the integrated dataset there were four upregulated pathways (ECM-Receptor Interaction, Cell Adhesion, Cell Communication and p53 Signaling), three of which deal with extracellular processes and six downregulated pathways, all of which deal with metabolism and metabolite degradation. The same results were found when doing GSEA on each of the single studies separately.

\section{Discussion}

The present microarray meta analysis makes use of the latest methods for microarray data integration and classification. Nevertheless, meta-analysis of microarray data still poses a challenge, mainly because researchers ask at least partly different questions and hence use different experimental designs. Moreover, the number of thyroid tumor microarray data available to researchers to date is still comparably low (compared to breast cancer, e.g.). Therefore, when doing meta analysis one is forced to use all data available, even if the patient cohorts represent a rather heterogeneous and potentially biased population. More specifically, it is difficult to obtain a homogenous collection of control material (from healthy patients). These are usually taken from patients who were operated for other thyroid disease which is in turn very likely to cause a change in gene expression as measured on microarrays. In other studies, healthy tissue surrounding the nodule was takes as control material. The generation of homogeneous patient cohorts is further hampered by limited availability of patient data like age, gender, genetic background, etc.

Nevertheless we believe to have shown that there still is a great deal of information to be discovered in the wealth of DNA microarray data deposited in databases like ArrayExpress and GEO [11,12]. As shown in the present and other papers $[9,13]$, methods for harvesting this information are available. Using approaches like DWD for data integration followed by classification and validation, it is possible to analyse a large number of samples at minimal cost. For the discovery of mRNAbased markers, a part of the scientific community has already moved on to other, more accurate, even higher parallel and even more expensive methods like mRNA sequencing using next-generation sequencing technologies. We (see Table 1) and others [14] have shown that feature selection in highly multivariate data like mircoarray data is not very robust, due to high feature numbers and low sample numbers. Applying these findings to next-generation sequencing data, it is likely that the feature selection problems will be even more pronounced, since feature numbers are even higher and even fewer people will be able to afford the analysis of sample sizes which account for the heterogeneous nature of clinical samples. This is why we believe that low cost/high sample number techniques like the one utilised here can provide valuable additional information.

This is also supported by another meta analysis on papillary thyroid carcinoma data which has been conducted by Fujarewicz et al. [15]. They used 3 datasets, two of which were used in our study as well (Jarzab and $\mathrm{He}$ ). They skipped data integration and used a bootstrap strategy instead, which consisted of iterative construction of Support Vector Machine (SVM) classifiers (on features selected for each iteration) based on randomly selected sets of specimen and testing the classifiers on the remaining samples. Through this sampling scheme, they select genes which exhibit little study bias and good discrimination between tumor subtypes. They achieved $98.5 \%$ classification accuracy between PTC and benign nodules on a 20 gene classification rule. 


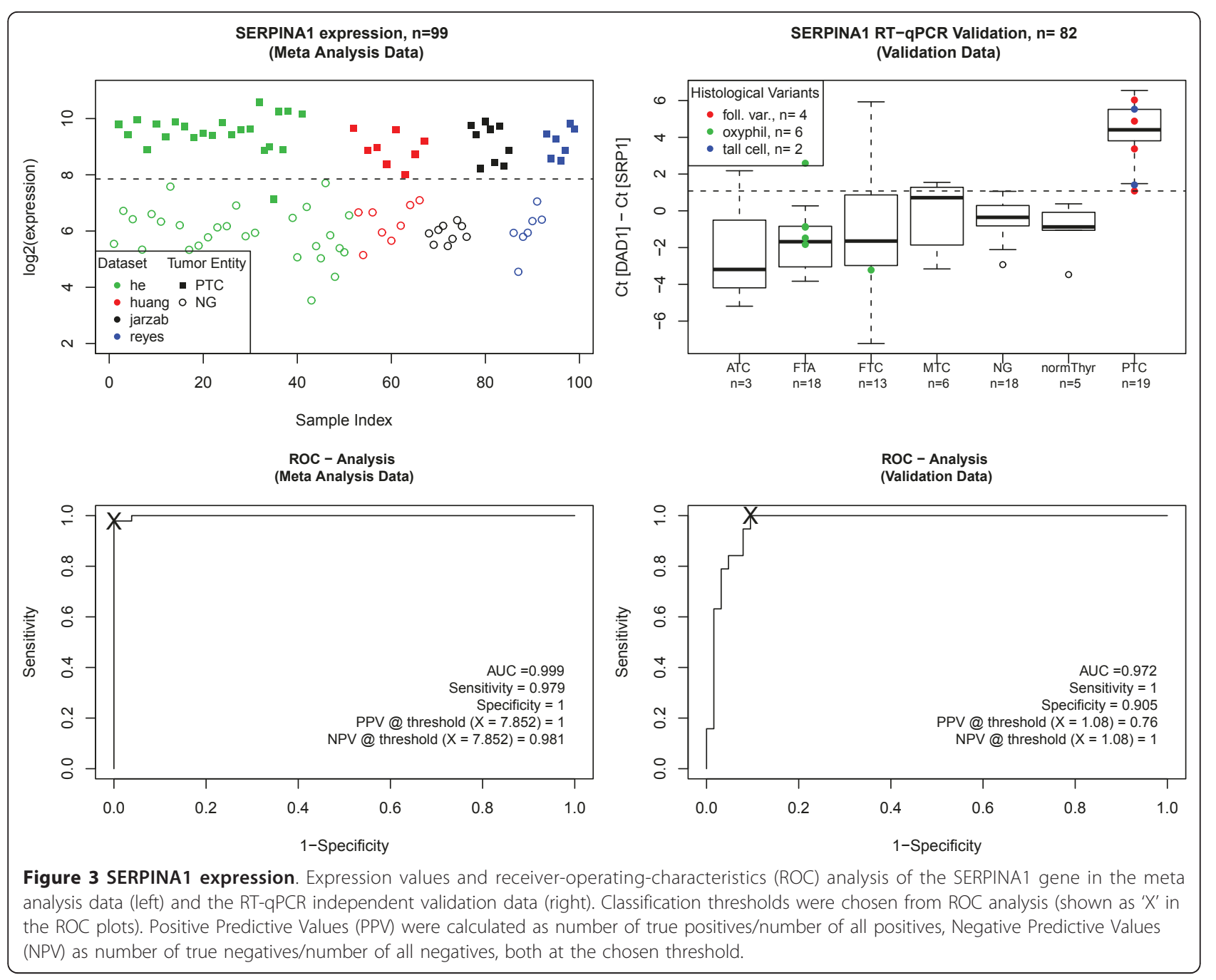

When doing meta analysis of microarray data, researchers have also based their approach on modeling of inter-study variation [16] or on comparing gene lists from published studies. A meta analysis and meta review by Griffith et. al. [17] has summarised genes with a diagnostic potential in the context of thyroid disease. They published lists of genes which appeared in more than one high-throughput study (Microarray, SAGE) analysing thyroid disease and applied a ranking system. In their analysis SER-PINA1 scored the third highest. Six of our top 20 most significant genes appear in the list of Griffith et al. Their approach is very useful, as one can include all studies in the analysis and is not limited to the studies where raw data is available. However, the studies generally follow very different analysis strategies, some more rigorous than others. It is not under the control of the meta-analyst how the authors arrived at the gene lists. It is also not possible to assign a measure of confidence at the gene level. However, given the overlap in the marker genes identified by these two methods, they seem to complement each other well for in-silico marker discovery. ANOVA-based approaches are very useful for identifying differentially regulated genes but to our knowledge there is no method to test these genes in the meta analysis data for their discriminatory power.

Most of these lists were generated from microarray analysis. However, even when comparing the genes in the classifiers to gene lists generated with independent technologies, like cDNA library generation as performed by Kaserer et al. [18], there is substantial overlap. SERPINA1 appears in their lists as well as seven of our top 20 genes.

SERPINA1 has been reported before as a potential diagnostic marker for papillary thyroid carcinoma in a study investigating its protein expression in thyroid biopsy tissue by Immunocytochemistry and Western Blot [19]. In line with our results, they found SERPINA1 immunoreactivity in nine of ten papillary biopsies while the surrounding tissue showed no such reaction. 
Table 2 Differential Expression Analysis

\begin{tabular}{|c|c|c|c|c|c|c|}
\hline SYMBOL & RefSeq & $\log \mathrm{FC}$ & adj.P.Val & Sens. & Spec. & KEGGID \\
\hline SERPINA1 & $\begin{array}{l}\text { NM_000295, NM_001002235, } \\
\text { NM_001002236 }\end{array}$ & 3.30 & $7.81 e-39$ & 0.98 & 1.00 & 04610 \\
\hline PROS1 & NM_000313 & 2.12 & $8.89 e-34$ & 0.98 & 1.00 & 04610 \\
\hline LRP4 & NM_002334 & 2.80 & $8.89 e-34$ & 1.00 & 0.96 & NA \\
\hline NPC2 & NM_006432 & 1.29 & $5.83 e-33$ & 1.00 & 0.94 & 04142 \\
\hline LAMB3 & NM_000228, NM_001017402 & 2.46 & $5.11 e-31$ & 0.96 & 1.00 & $04510,04512,05200,05222$ \\
\hline DPP4 & NM_001935 & 2.86 & $6.96 e-31$ & 0.98 & 0.98 & NA \\
\hline SDC4 & NM_002999 & 1.68 & $5.50 e-30$ & 0.96 & 0.96 & 04512,04514 \\
\hline IPCEF1 & NM_015553 & -2.10 & $2.88 \mathrm{e}-29$ & 1.00 & 0.00 & NA \\
\hline QPCT & NM_012413 & 2.09 & $3.00 e-29$ & 0.98 & 1.00 & NA \\
\hline MPPED2 & NM_001584 & -2.28 & 7.73e-29 & 1.00 & 0.00 & NA \\
\hline TIMP1 & NM_003254 & 1.85 & 1.48e-27 & 0.96 & 0.94 & NA \\
\hline TFF3 & NM_003226 & -3.42 & $2.07 e-27$ & 1.00 & 0.00 & NA \\
\hline PRSS23 & NM_007173 & 1.48 & $2.32 e-27$ & 0.98 & 0.98 & NA \\
\hline MET & NM_000245 & 1.64 & $2.37 e-27$ & 0.94 & 0.98 & $\begin{array}{l}\text { 04060, 04144, 04360, 04510, 04520, 05120, 05200, 05210, } \\
05211,05218\end{array}$ \\
\hline $\mathrm{CDH} 3$ & NM_001793 & 2.72 & $3.45 e-27$ & 0.96 & 0.94 & 04514 \\
\hline GGCT & NM_024051 & 1.21 & $2.74 \mathrm{e}-26$ & 0.98 & 0.92 & 00480 \\
\hline PDLIM4 & NM_003687 & 2.21 & $3.37 e-26$ & 0.96 & 0.98 & NA \\
\hline KRT19 & NM_002276 & 2.33 & $3.40 e-25$ & 0.98 & 0.98 & NA \\
\hline CITED1 & NM_004143 & 3.17 & $4.42 e-25$ & 0.94 & 0.96 & NA \\
\hline CHI3L1 & NM_001276 & 3.50 & $4.42 e-25$ & 0.96 & 0.90 & NA \\
\hline
\end{tabular}

The first 20 entries in the toptable of differential expression in meta analysis data, including log2 fold changes (logFC) and Benjamini-Hochberg adjusted p-values (adj.P.Value) as calculated by the limma-software. Sensitivity and Specificity are given for the distinction of PTC vs NG at maximum accuracy. Annotational information like gene symbols, consensus sequence identifiers (RefSeq) and KEGG Pathway IDs are shown.

In GSEA, the most apparent feature of PTC is an upregulation of extracellular activities, like cell communication and adhesion and extracellular matrix receptor interactions. While the gene lists and the derived classification rules of the 4 studies under investigation here show little over-lap, we found the same pathways to be overrepresented when each of the single studies is being analysed separately. Upregulation of ECM microenvironment genes have been described as an effect of BRAF mutations through phospho-ERK1/2 signalling, which in turn may be involved in the up-regulation of some ECM remodeling genes like TSP-1 [20]. While ECM has been seen as mere scaffolding for many years, recently its role in tumor progression and invasion through ECM remodeling and stiffening of the tissue stroma became evident [21]. Similar Pathways have been reported to be implicated in the progression from simple ductal hyperplasia to atypical ductal hyperplasia and further to ductal carcinoma in situ in ER+ sporadic breast cancer by Emery et al. [22]. Like us, they found, amongst other pathways, overrepresentation of the ECM-receptor interaction, cell communication and p53 signaling pathways, but none of the downregulated metabolic pathways which we identified in papillary thyroid carcinoma. Their data also suggests, that in breast cancer progression, dysregulation of the ECM-epithelial cell interactions is an early event, even before any evidence of invasion becomes visible. Similarly, Birnie et al. [23] reported Cell Adhesion and ECM receptor interactions pathway dysregulated when comparing prostate cancer stem cells $\left(C D 133^{+} / \alpha_{2} \beta_{1}^{\text {high }}\right)$ to their normal and differentiated counterparts $\left(C D 133^{-} / \alpha_{2} \beta_{1}^{\text {low }}\right)$.

The power of the meta analysis approach adopted here is demonstrated by a $99 \%$ loocvaccuracy $(97.9 \%$ weighted average accuracy in the study crossvalidation) for the distinction between papillary thyroid carcinoma and benign nodules. One sample was classified wrongly, and although it is not possible to correctly map the samples from this analysis to the original analysis [7], the misclassified sample is from the same group (PTC, validation group) as the sample which was wrongly classified in the original analysis. According to Jarzab et. al. the sample was an outlier because it contained only $\approx$ $20 \%$ tumor cells.

In the qPCR data we achieved a prediction accuracy of $93 \%$. We chose the threshold to allow for false positives rather than false negatives. For this threshold 6 samples were false positives and none false negative. Three follicular nodules were false positive, one of them was a recurrent FTC nodule and one was an oxyphilic FTA nodule. The third was a classical FTC. Two 
MTC and one ATC nodules were falsely positive. The latter two entities are rather easy to distinguish by alternative methods like cytology or serum calcitonin testing (MTC). However, the raw, unnormalised data (not shown) suggests, that the slightly elevated levels in the MTC and ATC group may not be down to elevated SER-PINA1 levels, but to a different cellular turnover in these cells which leads to a problem in the normalisation procedure. A more thorough search for appropriate normalisation genes may circumvent this problem.

Gerhard et al. [24] studied the intra- and inter-observer variability in the cytological assessment of fine needle aspirates from thyroid nodules. They found $24 \%$ (n = 97) disagreement between two observers, both of them experienced pathologists. While most of these disagreements were between different follicular nodules, there was still an inter-observer disagreement of $6.3 \%$ for the PTC nodules. This highlights the need for objective diagnostic methods which include the possibility to assign a measure of confidence in the final diagnosis. While PTC shows the most distinct cytological features and is therefore relatively easy to diagnose, a molecular diagnostic test could also provide the possibility to include molecular markers for tumor entities which are harder to diagnose by cytology.

\section{Conclusions}

These results are very encouraging, however, more studies need to be carried out in order to be able to transfer these findings into clinical applications for routine diagnostics. Firstly, markers with sufficient sensitivity and specificity for the other histological subtypes need to be found. There are few studies reporting molecular classifiers for follicular thyroid disease [25,26], and these classifiers cannot be applied to data from other studies (data not shown). To date, microarray studies for medullary and anaplastic thyroid carcinoma are largely missing. Secondly, in all of these studies, frozen thyroid nodules e.g. from instantaneous section pathology have been used. However, molecular markers for thyroid carcinomas will only be useful for routine diagnostics, if they exhibit the same discriminative power in fine needle aspiration biopsy (FNAB) samples. Microarray data from a study on thyroid FN-ABs [27] and a study on FNABs of various different tumor types [28] show good correlation between the aspirate and the tissue, but more detailed data is needed.

\section{Methods}

\section{Datasets}

Datasets were downloaded either from websites or from public repositories (GEO, ArrayExpress, see Table 3).

\section{Gene Mapping}

The first step in any meta analysis of microarray data is to find the set of genes which is shared by all microarray platforms used in the analysis. Traditionally, overlap is assessed by finding common Entrez-Gene or UniGene (National Center for Biotechnology Information, http:// www.ncbi.nlm.nih.gov/) identifiers. This, however, disregards all possible splice variations in the genes under investigation. For example, if a gene had 2 splice variants, one of which was Differentially expressed in the experiment and the other not and if one platform would contain an oligo specific only to the Differentially expressed variant and the other platform only an oligo to the other variant, then a matching based on UniGene would merge probes which measure different things.

To overcome this problem, the approach adopted here merges only probes which annotate to the same set of RefSeq identifiers. To this end all matching RefSeqs were downloaded for each probe(set) via the Bioconductor annotation packages (hgu133a, hgu95a and hgu133plus2; available at http://www.bioconductor.org). Probes which annotate to the same set of RefSeq identifiers were deemed to be similar. The median value was used, if one set of RefSeqs was represented by multiple probes on the array. 5707 different sets of RefSeqs were present on all arrays.

\section{Preprocessing and Data Integration}

First each dataset was background-corrected and normalised separately, as recommended for each platform $[29,30]$, then they were merged and quantile normalised collectively. Despite all preprocessing, it has been shown that data generated in different labs or on different microarray platforms or on different generations of the same platform may not be comparable due to platform or lab specific biases [8]. This is also evident from principal component analysis and hierarchical clustering of the merged data as shown in Figure 1. In order to correct for these biases, methods have been developed for integration of microarray data. One of these methods is Distance Weighted Discrimination (DWD) which is described in detail elsewhere [9]. Briefly, DWD projects

Table 3 Datasets used for meta analysis

\begin{tabular}{rllllll}
\hline \multicolumn{1}{c}{ Published } & PTC & o.d. & c.lat & Platform & Size \\
\hline He PNAS 2005 & 9 & 0 & 9 & Affy U133plus & $54 \mathrm{k}$ \\
Huang & PNAS 2001 & 8 & 0 & 8 & Affy U95A & $12 \mathrm{k}$ \\
Jarzab Cancer Res 2005 & 23 & 11 & 17 & Affy U133A & 22k \\
Reyes & not published & 7 & 0 & 7 & Affy U133A & 22k \\
\hline
\end{tabular}

Microarray Data used for meta analysis. The studies used 2 types of benign samples: samples from patients that were operated for other thyroid disease (o.d) and samples from the contralateral lobe (c.lat). Data was obtained from GEO http://www.ncbi.nlm.nih.gov/geo/ or institutional websites. 
data points onto the normal vector of a class (dataset) separating hyperplane as calculated by a modified Support Vector Machine (SVM) and sub-tracts the class (dataset) means. Therefore, for a multi-class problem (more than 2 datasets to merge), the datasets need to be merged sequentially. For 4 datasets this leads to 24 different possibilities for merging, not including tree structured approaches, e.g instead of $(((1+2)+3)+4)$, consider $((1+2)+(3+4))$. The merging orders applied here were chosen on the general idea that similar and larger datasets should be merged first and more disparate ones later (personal communication). It is also worth noting, that adding a sample to a DWD merged dataset will change the whole dataset just like adding a new number to a vector of numbers will change its mean.

\section{Classification}

For probe selection, classification and crossvalidation a nearest shrunken centroid method was chosen [31] (implemented in the Bioconductor package pamr). Briefly, it calculates several different possible classifiers using different shrinkage thresholds (i.e. different number of genes) and finds the best threshold from crossvalidation. Here we picked the classifier with the smallest number of genes (largest threshold), if more than one threshold yielded the same crossvalidation results.

\section{External Validation}

Thyroid nodules were obtained from instantaneous section pathology at the Vienna General Hospital with the approval of the local ethics committee. We analysed 82 thyroid samples of 7 different entities: ATC $(\mathrm{n}=3)$, FTA $(\mathrm{n}=18)$, FTC $(\mathrm{n}=13), \operatorname{MTC}(\mathrm{n}=6)$, normal thyroid ( $\mathrm{n}$ $=5)$, PTC $(\mathrm{n}=19)$ and NG $(\mathrm{n}=18)$. The choice of nodules also included a high rate of histological variants, like follicular variant PTC, tall cell PTC and oxyphilic follicular nodules. Approximately $30 \mathrm{mg}$ of tissue was lysed in RLT-buffer on a FastPrep FP120 instrument (Qbiogene p/n 6001-120) and extracted using Qiagen All-Prep DNA/RNA extraction (Qiagen p/n 80204) according to manufacturers instructions. RNA quality was assessed on the Agilent 2100 Bioanalyser $(\mathrm{p} / \mathrm{n}$ G2938C).

We had chosen following ABI TaqMan Assays: ACTB (Hs99999903_m1), CASC3 (Hs00201226_m1), DAD1 (Hs00154671_m1), PPIA (Hs99999904_m1) and SerpinA1 (Hs01097800_m1). Oligo-dT primed reverse transcription was done using Superscript III (Invitrogen), following the suppliers instructions. RT reaction with 180 ng RNA was used for three controls (ACTB, DAD1, PPIA) and the SerpinA1 gene in triplicates for quantitative PCR (12 reactions). We used $125 \mu \mathrm{l}$ of the ABI $2 \times$ Gene-Expression-Mastermix, $20 \mu \mathrm{l}$ cDNA and 42,5 $\mu \mathrm{l}$
RNase free water for the PCR mastermix. Aliquots of 15 $\mu \mathrm{l}$ (for 4 assays in triplicates) were added to a $1 \mu l+4$ $\mu \mathrm{l}$ dilution of each assay $(5 \mu \mathrm{l}$ diluted assay plus $15 \mu \mathrm{l}$ cDNA Mastermix). Cycling conditions were $2 \sec 50^{\circ} \mathrm{C}$, $10 \sec 95^{\circ} \mathrm{C}$ and then $15 \sec 95^{\circ} \mathrm{C}, 1$ sec $60^{\circ} \mathrm{C}$ for 50 cycles (ABI 5700 Sequence Detection System). Using the method of Vandesompele et.al [32], DAD1 was picked as the gene with the highest stability and therefore used as housekeeping gene for normalisation.

\section{Gene Set Enrichment Analysis (GSEA)}

First, inference statistics was calculated using the bioconductor package limma [33]. This includes non-specific filtering and linear modelling using empirical bayes moderated variances and Benjamini-Hochberg correction for multiple testing. For each gene, limma calculates a log odds ratio for differential expression (Bvalue). GSEA for pathway enrichment was performed on Students t-statistics using the geneSetTest function in the limma package. All data analysis except DWD-integration (Matlab ${ }^{\circledR}$, Natick, MA) was done in R/bioconductor http://www.bioconductor.org [34]. R-codes and qPCR raw data is available at http://www.methcancerdb. net/methcancerdb/img/ThyroidMetaAnalysis.zip.

\section{Author details}

${ }^{1}$ Molecular Medicine, AIT - Austrian Institute of Technology, A-1190 Vienna, Austria. ${ }^{2}$ Department of Clinical Pathology, University of Vienna Medical School, A-1090 Vienna, Austria. Institute of Applied Statistics and

Computing, University of Natural Ressources and Life Sciences, 1190 Vienna, Austria.

\section{Authors' contributions}

$\mathrm{KV}, \mathrm{CN}, \mathrm{KK}$ and FL carried out the conception and design of the study, statistical analysis was carried out by KV under supervision of $\mathrm{FL}$, conception and completion of molecular analysis was done by MM, collection and assessment of clinical material and data was done by KK and OK. KV prepared the manuscript including critical intellectual input and revisions by all authors. All authors read and approved the final manuscript.

\section{Competing interests}

The authors declare that they have no competing interests.

Received: 3 November 2010 Accepted: 6 April 2011

Published: 6 April 2011

\section{References}

1. Mazzaferri $\mathrm{E}$ : Thyroid cancer in thyroid nodules: finding a needle in the haystack. Am J Med 1992, 93:359-362.

2. Cooper DS, Doherty GM, Haugen BR, Kloos RT, Lee SL, Mandel SJ, Mazzaferri EL, Mclver B, Sherman SI, Tuttle RM: Management guidelines for patients with thyroid nodules and differentiated thyroid cancer. Thyroid 2006, 16(2):109-142.

3. Ravetto C, Colombo L, Dottorini M: Usefulness of fineneedle aspiration in the diagnosis of thyroid carcinoma: a retrospective study in 37,895 patients. Cancer 2000, 90:357-363.

4. Chang H, Lin J, Chen J, Huang B, Hsueh C, Jeng L, Tsai J: Correlation of fine needle aspiration cytology and frozen section biopsies in the diagnosis of thyroid nodules. J Clin Pathol 1997, 50:1005-1009.

5. van't Veer LJ, Dai H, van de Vijver MJ, He YD, Hart AAM, Mao M, Peterse HL, van der Kooy K, Marton MJ, Witteveen AT, Schreiber GJ, Kerkhoven RM, Roberts C, Linsley PS, Bernards R, Friend SH: Gene expression profiling 
predicts clinical outcome of breast cancer. Nature 2002, 415(6871):530-536.

6. van de Vijver MJ, He YD, van't Veer LJ, Dai H, Hart AAM, Voskuil DW, Schreiber GJ, Peterse JL, Roberts C, Marton MJ, Parrish M, Atsma D, Witteveen A, Glas A, Delahaye L, van der Velde T, Bartelink H, Rodenhuis S, Rutgers ET, Friend SH, Bernards R: A geneexpression signature as a predictor of survival in breast cancer. N Engl J Med 2002, 347(25):1999-2009.

7. Jarzab B, Wiench M, Fujarewicz K, Simek K, Jarzab M, OczkoWojciechowska M, Wloch J, Czarniecka A, Chmielik E, Lange D, Pawlaczek A, Szpak S, Gubala E, Swierniak A: Gene expression profile of papillary thyroid cancer: sources of variability and diagnostic implications. Cancer Res 2005, 65(4):1587-1597.

8. Eszlinger M, Wiench M, Jarzab B, Krohn K, Beck M, Lauter J, Gubala E, Fujarewicz K, Swierniak A, Paschke R: Meta- and reanalysis of gene expression profiles of hot and cold thyroid nodules and papillary thyroid carcinoma for gene groups. J Clin Endocrinol Metab 2006, 91(5):1934-1942.

9. Benito M, Parker J, Du Q, Wu J, Xiang D, Perou CM, Marron JS: Adjustment of systematic microarray data biases. Bioinformatics 2004, 20:105-114.

10. Subramanian A, Tamayo P, Mootha VK, Mukherjee S, Ebert BL, Gillette MA, Paulovich A, Pomeroy SL, Golub TR, Lander ES, Mesirov JP: Gene set enrichment analysis: a knowledge-based approach for interpreting genome-wide expression profiles. Proc Natl Acad Sci USA 2005, 102:15545-15550.

11. Parkinson H, Kapushesky M, Shojatalab M, Abeygunawardena N, Coulson R, Farne A, Holloway E, Kolesnykov N, Lilja P, Lukk M, Mani R, Rayner T, Sharma A, William E, Sarkans U, Brazma A: ArrayExpress-a public database of microarray experiments and gene expression profiles. Nucleic Acids Res 2007, 35:D747-750

12. Edgar R, Domrachev M, Lash AE: Gene Expression Omnibus: NCBI gene expression and hybridization array data repository. Nucleic Acids Res 2002, 30:207-210.

13. Lu Y, Lemon W, Liu PY, Yi Y, Morrison C, Yang P, Sun Z, Szoke J, Gerald WL, Watson M, Govindan R, You M: A gene expression signature predicts survival of patients with stage I non-small cell lung cancer. PLoS Med 2006, 3:e467.

14. Ambroise C, McLachlan GJ: Selection bias in gene extraction on the basis of microarray gene-expression data. Proc Natl Acad Sci USA 2002, 99:6562-6566.

15. Fujarewicz K, Jarzab M, Eszlinger M, Krohn K, Paschke R, OczkoWojciechowska M, Wiench M, Kukulska A, Jarzab B, Swierniak A: A multigene approach to diffierentiate papillary thyroid carcinoma from benign lesions: gene selection using support vector machines with bootstrapping. Endocr Relat Cancer 2007, 14:809-826.

16. Choi JK, Yu U, Kim S, Yoo OJ: Combining multiple microarray studies and modeling interstudy variation. Bioinformatics 2003, 19(Suppl 1):84-90.

17. Griffith OL, Melck A, Jones SJM, Wiseman SM: Meta-analysis and metareview of thyroid cancer gene expression profiling studies identifies important diagnostic biomarkers. J Clin Oncol 2006, 24(31):5043-5051.

18. Kaserer K, Knezevic V, Pichlhofer B, Scheuba C, Passler C, Worth J, Niederle B, Krizman D: Construction of CDNA libraries from microdissected benign and malignant thyroid tissue. Lab Invest 2002, 82(12):1707-1714.

19. Poblete M, Nualart F, del Pozo M, Perez J, Figueroa C: Alpha 1-antitrypsin expression in human thyroid papillary carcinoma. Am J Surg Pathol 1996, 20:956-963.

20. Nucera C, Porrello A, Antonello ZA, Mekel M, Nehs MA, Giordano TJ, Gerald D, Benjamin LE, Priolo C, Puxeddu E, Finn S, Jarzab B, Hodin RA, Pontecorvi A, Nose V, Lawler J, Parangi S: B-Raf(V600E) and thrombospondin-1 promote thyroid cancer progression. Proc Natl Acad Sci USA 2010, 107:10649-10654.

21. Erler JT, Weaver VM: Three-dimensional context regulation of metastasis. Clin Exp Metastasis 2009, 26:35-49.

22. Emery LA, Tripathi A, King C, Kavanah M, Mendez J, Stone MD, de las Morenas A, Sebastiani P, Rosenberg CL: Early dysregulation of cell adhesion and extracellular matrix pathways in breast cancer progression. Am J Pathol 2009, 175:1292-1302.

23. Birnie R, Bryce SD, Roome C, Dussupt V, Droop A, Lang SH, Berry PA, Hyde CF, Lewis JL, Stower MJ, Maitland NJ, Collins AT: Gene expression profiling of human prostate cancer stem cells reveals a pro- inflammatory phenotype and the importance of extracellular matrix interactions. Genome Biol 2008, 9:R83.

24. Gerhard R, da Cunha Santos G: Inter- and intraobserver reproducibility of thyroid fine needle aspiration cytology: an analysis of discrepant cases. Cytopathology 2007, 18:105-111

25. Weber F, Shen L, Aldred MA, Morrison CD, Frilling A, Saji M, Schuppert F, Broelsch CE, Ringel MD, Eng C: Genetic classification of benign and malignant thyroid follicular neoplasia based on a three-gene combination. J Clin Endocrinol Metab 2005, 90(5):2512-2521.

26. Lacroix L, Lazar V, Michiels S, Ripoche H, Dessen P, Talbot M, Caillou B, Levillain JP, Schlumberger M, Bidart JM: Follicular thyroid tumors with the PAX8-PPARgamma1 rearrangement display characteristic genetic alterations. Am J Pathol 2005, 167:223-231.

27. Lubitz C, Ugras S, Kazam J, Zhu B, Scognamiglio T, Chen Y, Fahey T: Microarray analysis of thyroid nodule fine-needle aspirates accurately classifies benign and malignant lesions. J Mol Diagn 2006, 8:490-498,

28. Centeno B, Enkemann S, Coppola D, Huntsman S, Bloom G, Yeatman T: Classification of human tumors using gene expression profiles obtained after microarray analysis of fine-needle aspiration biopsy samples. Cancer 2005, 105:101-109

29. Bolstad BM, Irizarry RA, Astrand M, Speed TP: A comparison of normalization methods for high density oligonucleotide array data based on variance and bias. Bioinformatics 2003, 19(2):185-193.

30. Smyth GK, Speed T: Normalization of CDNA microarray data. Methods 2003, 31(4):265-273.

31. Tibshirani R, Hastie T, Narasimhan B, Chu G: Diagnosis of multiple cancer types by shrunken centroids of gene expression. Proc Natl Acad Sci USA 2002, 99(10):6567-6572.

32. Vandesompele J, De Preter K, Pattyn F, Poppe B, Van Roy N, De Paepe A, Speleman F: Accurate normalization of real-time quantitative RT-PCR data by geometric averaging of multiple internal control genes. Genome Biol 2002, 3(7)

33. Smyth GK: Linear models and empirical bayes methods for assessing differential expression in microarray experiments. Stat Appl Genet Mol Biol 2004, 3:Article3.

34. Gentleman R, Carey V, Bates D, Bolstad B, Dettling M, Dudoit S, Ellis B, Gautier L, Ge Y, Gentry J, Hornik K, Hothorn T, Huber W, lacus S, Irizarry R, Leisch F, Li C, Maechler M, Rossini A, Sawitzki G, Smith C, Smyth G, Tierney L, Yang J, Zhang J: Bioconductor: open software development for computational biology and bioinformatics. Genome Biol 2004, 5:R80.

\section{Pre-publication history}

The pre-publication history for this paper can be accessed here: http://www.biomedcentral.com/1755-8794/4/30/prepub

\section{doi:10.1186/1755-8794-4-30}

Cite this article as: Vierlinger et al.: Identification of SERPINA1 as single marker for papillary thyroid carcinoma through microarray meta analysis and quantification of its discriminatory power in independent validation. BMC Medical Genomics 2011 4:30

\section{Submit your next manuscript to BioMed Central and take full advantage of:}

- Convenient online submission

- Thorough peer review

- No space constraints or color figure charges

- Immediate publication on acceptance

- Inclusion in PubMed, CAS, Scopus and Google Scholar

- Research which is freely available for redistribution

Submit your manuscript at www.biomedcentral.com/submit
C Biomed Central 